\title{
Pediatric Triage Variations Among Nurses, Pediatric and Emergency Residents Using the Canadian Triage and Acuity Scale
}

Saleh Alshaibi ( $\square$ alshaibi213@gmail.com )

King Abdulaziz Medical City https://orcid.org/0000-0003-3697-2867

\section{Tala AlBassri}

King Saud bin Abdulaziz University for Health Sciences College of Medicine

\section{Suliman AlQeuflie}

King Abdulaziz Medical City

Winnie Philip

King Saud bin Abdulaziz University for Health Sciences

Nesrin Alharthy

King Abdulaziz Medical City

Research article

Keywords: CTAS, Triaging system, Pediatrics

Posted Date: February 23rd, 2021

DOl: https://doi.org/10.21203/rs.3.rs-37889/v2

License: (c) (1) This work is licensed under a Creative Commons Attribution 4.0 International License. Read Full License

Version of Record: A version of this preprint was published at BMC Emergency Medicine on November 22nd, 2021. See the published version at https://doi.org/10.1186/s12873-021-00541-0. 


\section{Abstract}

\section{Background}

Emergency care continues to be a challenge, since patients' arrival is unscheduled and could occur at the same time which may fill the Emergency Department with non-urgent patients. Triaging is an integral part of every busy ED. The Canadian Triage and Acuity Scale (CTAS) is considered to accurate tool to be used outside Canada. This study aims to identify the chosen triage level and compare the variation between registered nurses, pediatric and adult emergency residents by using CTAS cases.

\section{Method}

This study was conducted at King Abdulaziz Medical City, Saudi Arabia. A cross-sectional selfadministered questionnaire was used, and which contains 15 case scenarios with different triage levels. All cases were adopted from a Canadian triage course after receiving permission. Each case provides the patient's symptoms, clinical signs and mode of arrival to the ED. The participants were instructed to assign a triage level using the following scale: $1=$ resuscitation, $2=$ =emergent, $3=$ =urgent, $4=$ less urgent, and $5=$ non-urgent. A non-random sampling technique was used for this study.

\section{$\underline{\text { Result }}$}

A total of 151 participants completed the study questionnaire. $73(48.34 \%)$ were nurses and $78(51.66 \%)$ were residents (42 pediatric residents and 36 emergency residents).

Each participant was given a score based on the correct answer for the case scenario. A mean score was compared among the nurses, pediatric residents and emergency residents. Since our data were not normally distributed, we used the Kruskal-Wallis test to observe statistical differences between the groups. The Kruskal Wallis Post-Hoc test was performed to see which groups were statistically significant, and it was found that there was a significant difference between nurses and pediatrics residents ( $P$ value $<0.001)$. Moreover, there were no significant differences found between nurses and ER residents $(P$ value $>0.05)$.

\section{Conclusion}

The triaging system was found to be a very important tool to prioritize patients based on their complaints. The results showed that nurses had the greatest experience in implementing patients on the right triage level. On the other hand, ER and pediatric residents demonstrated a need to develop more knowledge about CTAS and become exposed more to the triaging system during their training.

\section{Background}

The emergency department (ED) is one of the most crucial sectors of the healthcare system. Emergency care continues to be a challenge, since patients' arrival is unscheduled with different disease acuity. 
There has been a steady increase in the number of patients visiting EDs annually, which leads to a rise in resource utilization. Studies have shown that about $25-60 \%$ of the patients seeking care in emergency departments are real emergency cases.(1)(2) However, EDs may become filled with non-urgent patients, leading to overcrowding. Overcrowded EDs affect the patient in terms of resources and adequate care in a timely fashion due to overwhelming ER staff physicians. (3) Furthermore, prioritizing and making a decisive decision about the severity of a condition will allow allocation of resources to sick patients in timely manner .(4)

Triage is a French word originating in the late 1700s, which means "to sort" or "to select ". In the past, it was used to sort injured soldiers by prioritizing them according to the type and urgency of their conditions.(5)(6) Today, triaging is an integral part of every busy emergency department. It ensures that patients receive appropriate attention in response to their clinical needs.

Over several years, many formalized systems appeared, which are used differently worldwide. These include the Australian Triage Scale (ATS), the Canadian Triage and Acuity Scale (CTAS), the Manchester Triage System (MTS), and the Emergency Severity Index (ESI).(7) In Saudi Arabia, there is still no unified triage system. However, CTAS has been applied for more than 10 years in different hospitals in Saudi Arabia, including King Abdullah Specialist Children's Hospital (KASCH).(8) Although the Canadian Triage and Acuity Scale (CTAS) was first utilized in Canada, several studies showed that CTAS is considered an accurate and reliable tool for patient assessment outside of Canada. It demonstrates reliability and validity in both children and adults.(9)(10) According to CTAS, triage is classified into five levels: $1=$ resuscitation, 2 = emergent, 3 = urgent, 4 = less urgent, 5 = non-urgent.(8) Determining the CTAS level is a combination of selecting the Canadian Emergency Department Information System (CEDIS) presenting a complaint and looking critically at the patient, with the information given from the patient, to assess whether he is stable. It will allow the nurse to choose appropriate modifiers to assign the most suitable CTAS level.(11)

During a short assessment, nurses identify signs and symptoms that determine the patient's urgency. The role of triaging nurses is vital for assessing the patient, such as communicating with the public, communicating with health professionals, assigning resources, initiating treatment protocols/first aid measures, monitoring and reassessing, participating in patient flow, and documenting.(12) A study conducted among nurses with at least six months of triage experience showed that there is moderate to low agreement among nurses in determining the level of triage for pediatric patients.(5) After a short assessment, physicians see patients in order of their urgency level and manage them accordingly. Also, there are some differences between physicians in triaging experience based on their specialties. This has been shown in several areas, including the management of febrile seizure and sedation use.(13) However, many studies have focused only on the management of cases, and not on physicians' or nurses' perceptions of triaging patients.(14)

Limited studies have been conducted in pediatrics triaging systems, specifically CTAS. Most previous studies have focused on adult populations or on different triaging systems that are not used in Saudi 
Arabia.(15) The objective of this study is to determine whether differences exist between pediatric and adult nurses and triaging in pediatric patients.

\section{Methods}

A cross-sectional design using a questionnaires survey to assess participants knowledge about patient triage using clinical scenarios in a period between 2018-2020. This study was conducted at King Abdulaziz Medical City, Saudi Arabia. A non-probability convenience sample was used, as we were conducting the study data collection during a clinical shift, and the responders were therefore selected based on their accessibility and time. Based on an estimate, we have around 80 residents in both emergency and pediatric residency programs. We targeted all, since we expected a response rate of around $80 \%$. Nurses were selected based on the known population size of 200 and $5 \%$ as a margin of error with a $95 \%$ confidence interval, and $50 \%$ as the response distribution, and the calculated sample size of 132. A rough estimate of 200 participants was considered for the study. All residents were included from general pediatrics and the adult emergency training program. Pediatrics and adult emergency nurses were also included. Although, Residents are not allowed to perform triage in our institute and they must be supervised by senior nurses but they get some clinical training during their ER rotations. We excluded emergency fellows and emergency consultants from the study due to expected high knowledge, which would have affected the study results.

A questionnaire of 15 case scenarios was distributed among nurses and residents. All cases were adopted from the CTAS course. Permission was obtained from the post-graduate training center and CATS course developer in order to use the material. The cases were reviewed and readjusted culturally and environmentally. Moreover, cases that could be triaged into two different levels were eliminated to avoid confusion. We included two to three from each level to reflect reality.

The case scenarios described patients coming to the ED. Each case provided background information on the patients, symptoms, important clinical signs, and mode of arrival to the ED. The participants were instructed to assign a triage level using the following scale: 1 = resuscitation, 2 = emergent, $3=$ urgent, $4=$ less urgent, and $5=$ non-urgent (Table 1). All the data collected were entered using Microsoft Excel. The data were analyzed using SPSS version 22 software. Since the data were not normally distributed, Kruskal Wallis test was used to determine the statistically significant differences between the three groups. A p-value of less than 0.05 was considered statistically significant. Results were expressed in tables and figures. Mean and Standard Deviation (SD) were used for continuous variables. The rates of agreement between pediatrics, adult residents and nurses were calculated using kappa statistics (weighted kappa) $(95 \% \mathrm{Cl})$. The following guidelines for the interpretation of kappa statistics were used: less than 0.40 , poor to fair; 0.41 to 0.60 , moderate; 0.61 to 0.80 , substantial; and greater than 0.80 , almost perfect. 
Table 1

CTAS time objectives

\begin{tabular}{|llll|}
\hline $\begin{array}{l}\text { Triage level Time for } \\
\text { triage }\end{array}$ & $\begin{array}{l}\text { Time to nursing } \\
\text { assignment }\end{array}$ & $\begin{array}{l}\text { Time to nursing } \\
\text { reassessment }\end{array}$ & $\begin{array}{l}\text { Time to physician } \\
\text { assessment }\end{array}$ \\
\hline I & $\leq 10 \mathrm{~min}$ & Continuous care & Immediate \\
\hline II & & Every $15 \mathrm{~min}$ & $\leq 15 \mathrm{~min}$ \\
\hline III & & Every $30 \mathrm{~min}$ & $\leq 30 \mathrm{~min}$ \\
IV & & Every $60 \mathrm{~min}$ & $\leq 60 \mathrm{~min}$ \\
V & & Every $120 \mathrm{~min}$ & $\leq 120 \mathrm{~min}$ \\
\hline
\end{tabular}

\section{Results}

Of the 151 participants (73 Nurses, 36 ER residents and 42 pediatric residents) a 100\% response rate was achieved for all case scenarios. The mean $( \pm S D)$ age was $31.14( \pm 6.76)$ years with the majority, $66 \%$, being females. Among the 73 nurses, 29 (40\%) were working in pediatrics and $44(60 \%)$ were working with adults. For the ED residents, $36.2 \%$ were in level R2, $22.2 \%$ were in levels R1 and R4 and $19.4 \%$ were in level R3. Similarly for pediatric residents, a greater part of them, $50 \%$, were in level R4, while $23.8 \%$ were in $\mathrm{R} 2,21.4 \%$ were in $\mathrm{R} 3$ and $4.8 \%$ were in $\mathrm{R} 1$.

Among pediatric residents $38.1 \%$ had used the CTAS to triage pediatric patients. Fifty-five percent of the pediatric residents knew about CTAS scales. Of the pediatric residents, all had performed an emergency rotation $(42,100 \%)$. In addition, comparing cases answers between the 3 groups, showing that the nurses have the most corrected answers in all different triage levels as demonstrated in the graph. ( Fig. 1)

Triage scores were compared using the Kruskal Wallis test and were statistically significant with a $p$ value of 0.006 . The mean ranks for nurses, emergency residents and pediatric residents were $86.41,73.6$ and 59.96, respectively (Table 2). Figure 2 describes the Kruskal Wallis Post Hoc test and shows that the scores were statistically significant among pediatric residents (mean rank $=59.96$ ) and nurses (mean rank $=86.41$ ) with a $p$ value of 0.005 . The scores were statistically insignificant for pediatric residentsemergency residents ( $p$ value $=0.493$ ) and for emergency resident-nurses ( $p$ value $=0.435)$ (Fig. 2$)$. 
Table 2

Comparing the Triage score among Nurses, Pediatric residents and emergency Residents

\begin{tabular}{|c|c|c|c|c|}
\hline Participants & No. of subjects & Mean Rank & Test used \& Test Statistic & $P$ value \\
\hline Nurse & 73 & 86.41 & Kruskal Wallis Test & $0.006^{*}$ \\
\hline Emergency Resident & 36 & 73.60 & Chi square $=10.158$ & \\
\hline Pediatric Resident & 42 & 59.96 & & \\
\hline Total & 151 & & & \\
\hline
\end{tabular}

Weighted Kappa along with a 95\% confidence interval was used to find the level of agreement among nurses and residents based on the level of training. The overall agreement levels for residents were moderate, with the weighted Kappa as 0.438 . The level of agreement was observed to be good among the nurses, with weighted Kappa as 0.688. Among the pediatric residents (Weighted Kappa= -1.455) and emergency residents (Weighted Kappa $=-0.470$ ), the agreement level was observed to be poor (Table 3 ).

Table 3

Level of agreement among Nurses and Residents (Pediatric and Emergency) with Weighted Kappa and 95\% Cl

\begin{tabular}{|llll|}
\hline & No. of subjects & Weighted Kappa & $95 \% \mathrm{Cl}$ \\
\hline Residents & 78 & 0.438 & $(-0.282-0.832)$ \\
\hline Pediatric Residents & 42 & -1.455 & $(-2.375-0.932)$ \\
\hline Emergency Residents & 36 & -0.470 & $(-1.556-0.577)$ \\
\hline Nurses & 73 & 0.688 & $(-0.077-1.00)$ \\
\hline Nurses and Residents & 151 & 0.193 & $(-0.213-0.473)$ \\
\hline
\end{tabular}

\section{Discussion}

Triage is a very important tool in emergency departments, which requires the whole team to be aware of in order to standardize the care of health care providers while handling patients and promote proper utilization of resources. Non-urgent cases are estimated to be $9 \%-54 \%$ in the United States of America, and $25.5 \%-60 \%$ in Canada.(1)(16) A well-structured triaging system and multidisciplinary team recommended to be implemented in each emergency department for optimum patient care and crowd control. The level of agreement among nurses was satisfactory, which indicates the strength of training and experience in the field of patient triaging. In contrast, patient triaging was not an integral part of the resident training program, which was reflected in the study findings. 
The results showed $51.3 \%, 56.6 \%$, and $59.9 \%$ mis-triaged the cases based on the standard scenarios adopted from CTAS for nurses, Emergency residents, and pediatric residents, respectively, which may negatively impact patient care. ED overcrowding affects the hospital financially.(14) Over-triaging can have a huge impact on hospital resources allocation and timely patient care. A study conducted among pediatrics injured patients found that $61 \%$ of the patients who were considered to have a severe trauma were discharged from the pediatrics center in less than 24 hours.(17) On the other hand, under-triage can harm the patient by delaying management and putting the patient at risk of deterioration, especially for the pediatric age group, which might be accompanied by unclear signs of serious illness.(16)(17)

Inter-rater agreement using the CTAS scale has been studied for many years, mostly among nurses. Dallaire et al. compared nurses based on their experience and found a moderate agreement among them (weighted kappa of 0.44).(18) This finding contradicts our result, in which we compared a number of senior and junior nurses with different years of experience and in which practice found a good agreement between nurses, with a weighted kappa of 0.688 . However, our study's results were similar to those of Alquraini et al., which also found good agreement (weighted kappa of 0.770).(8) In addition, upon comparison between pediatrics residents, poor agreement was found (weighted kappa of -1.455) in this study. In contrast, a moderate agreement was concluded by Bergeron et al. (weighted kappa of 0.419).(5) In terms of physicians compared to nurses, this study resulted in a poor level of agreement (weighted kappa 0.193). Unlike our finding, a meta-analysis of 12 studies showed a pooled estimator of a good level of agreement with a value of 0.797.(19) Unsurprisingly, nurses performed better than residents in our study, due to their daily experience and regular practice of using a triaging scale.

This study had some limitations. First of all, we used paper-based case scenarios instead of real ED patients. Even though the cases in this study were adopted from the CTAS courses with some cultural modifications, few studies support that triage reliability testing can be tested using case scenarios as an alternative to real patients.(20) The data presented in this study comes from only one institution. This could affect the ability to generalize our findings to other facilities. Since our center is considered one the largest emergency trauma centers in the region, we believe that our setting is ideal for testing CTAS reliability. We also used a limited number of scenarios, and all of them were pediatrics populations. We specifically chose the more common scenarios seen in our region. Since adult Emergency residents mandatorily rotate under pediatric ED, we included only pediatric cases.

\section{Conclusion}

In conclusion, patients can present with a broad spectrum of emergencies that range from minor to severe presentations. In order to avoid overcrowding, having a well-structured triaging system is crucial to every ED. Also, nurses and physicians need to be more aware of how to use and apply this system. Our results showed that nurses had the highest level of experience in implementing patients on the right triage level, due to their knowledge and practice with CTAS scale. Additionally, these results signify the importance of emergency and pediatric residents to become familiar and have enough exposure to and training with the triaging system. 


\section{List Of Abbreviations}

ED

Emergency Department

ATS

Australian Triage Scale

CTAS

Canadian Triage and Acuity Scale

MTS

Manchester Triage System

ESI

Emergency Severity Index

$\mathrm{KASCH}$

King Abdullah Specialist Children's Hospital

CEDIS

Canadian Emergency Department Information System

SD

Standard Deviation

\section{Declarations}

Ethics approval and consent to participate:

This study was approved by the ethical committee of King Abdullah International Medical Research Center. All participants were informed about this study and written consent were obtained.

Consent for publication: Not applicable

Availability of data and materials: The datasets used and/or analyzed during the current study are available from the corresponding author on reasonable request

Competing interests: The authors declare that they have no competing interests

Funding: The authors did not receive any grant or funding support from any funding agencies in the public, commercial, or not-for-profit sectors in conducting this study.

Authors' contributions:

SS and TB conducted the study design, collected the data, rafting the article and revising it critically for important intellectual content. WP performed the statistical analysis. NH and SQ final approval of the version to be submitted. The author(s) read and approved the final manuscript.

Acknowledgements: Not applicable 


\section{References}

1. Ahmed N, Khan SS, Mehmood Y, Ahad B, Hussain H, Ullah N. HOSPITAL ADMINISTRATION A Study on the Factors Associated with Non- Urgent Utilization of Emergency Department. 2001; (October):83-9.

2. Eroglu SE, Toprak SN, Urgan O, Onur OE, Denizbasi A, Akoglu H, et al. Evaluation of non-urgent visits to a busy urban emergency department. Saudi Med J. 2012;33(9):967-72.

3. Lee JY, Oh SH, Peck EH, Lee JM, Park KN, Kim SH, et al. The validity of the Canadian Triage and Acuity Scale in predicting resource utilization and the need for immediate life-saving interventions in elderly emergency department patients. Scand J Trauma Resusc Emerg Med [Internet]. 2011;19(1):68. Available from: http://www.sjtrem.com/content/19/1/68.

4. Twomey M, Cheema B, Buys H, Cohen K, de Sa A, Louw P, et al. Vital signs for children at triage: A multicentre validation of the revised South African triage scale (SATS) for children. South African Med J. 2013;103(5):304-8.

5. Iserson KV, Moskop JC. Triage in Medicine, Part I: Concept, History, and Types. Ann Emerg Med. 2007;49(3):275-81.

6. Bergeron S, Gouin S, Bailey B, Patel H. Comparison of triage assessments among pediatric registered nurses and pediatric emergency physicians. Acad Emerg Med. 2002;9(12):1397-401.

7. Hinson JS, Martinez DA, Cabral S, George K, Whalen M, Hansoti B, et al. Triage Performance in Emergency Medicine: A Systematic Review. Ann Emerg Med [Internet]. 2019;74(1):140-52. Available from: https://doi.org/10.1016/j.annemergmed.2018.09.022.

8. Alquraini M, Awad E, Hijazi R. Reliability of Canadian Emergency Department Triage and Acuity Scale (CTAS) in Saudi Arabia. Int J Emerg Med [Internet]. 2015;8(1):6-9. Available from: http://dx.doi.org/10.1186/s12245-015-0080-5.

9. Elkum NB, Barrett CA, Al-Omran H. Canadian Emergency DepartmentTriage and Acuity Scale: Implementation in a tertiary care center in Saudi Arabia. BMC Emerg Med [Internet]. 2011;11(1):3. Available from: http://www.biomedcentral.com/1471-227X/11/3.

10. De Magalhães-Barbosa MC, Robaina JR, Prata-Barbosa A, Lopes CDS. Validity of triage systems for paediatric emergency care: A systematic review. Emerg Med J. 2017;34(11):711-9.

11. Bullard MJ, Musgrave E, Warren D, Unger B, Skeldon T, Grierson R, et al. Revisions to the Canadian Emergency Department Triage and Acuity Scale (CTAS) Guidelines 2016. Can J Emerg Med. 2017;19(S2):18-27.

12. Andersson AK, Omberg $M$, Svedlund $M$. Triage in the emergency department-a qualitative study of the factors which nurses consider when making decisions. Nurs Crit Care. 2006;11(3):136-45.

13. Van Veen M, Moll HA. Reliability and validity of triage systems in paediatric emergency care. Scand $J$ Trauma Resusc Emerg Med. 2009;17(1):1-8.

14. Chime NO, Katznelson J, Gangadharan S, Walsh B, Lobner K, Brown L, et al. Comparing practice patterns between pediatric and general emergency medicine physicians: A scoping review. Pediatr 
Emerg Care. 2017;33(4):278-86.

15. Of P, Emergency P, Visits R, King AT. Brief Reports PATTERNS OF PEDIATRIC EMERGENCY ROOM VISITS AT KING KHALID. 1997;17(3):360-2.

16. Wang L, Zhou H, Zhu JF. Application of the emergency severity index in pediatric emergency department. Chinese J Emerg Med. 2011;20(4):418-20.

17. Hewes HA, Christensen M, Taillac PP, Mann NC, Jacobsen KK, Fenton SJ. Consequences of pediatric undertriage and overtriage in a statewide trauma system. J Trauma Acute Care Surg. 2017;83(4):662-7.

18. Dallaire C, Poitras J, Aubin K, Lavoie A, Moore L. Emergency department triage: Do experienced nurses agree on triage scores? J Emerg Med. 2012;42(6):736-40.

19. Pishbin E, Ebrahimi M, Mirhaghi A. Do physicians and nurses agree on triage levels in the emergency department? A meta-analysis. Notfall Rettungsmedizin. 2019;22(5):379-85.

20. Worster A, Sardo A, Eva K, Fernandes CMB, Upadhye S. Triage Tool Inter-rater Reliability: A Comparison of Live Versus Paper Case Scenarios. J Emerg Nurs. 2007;33(4):319-23.

\section{Figures}

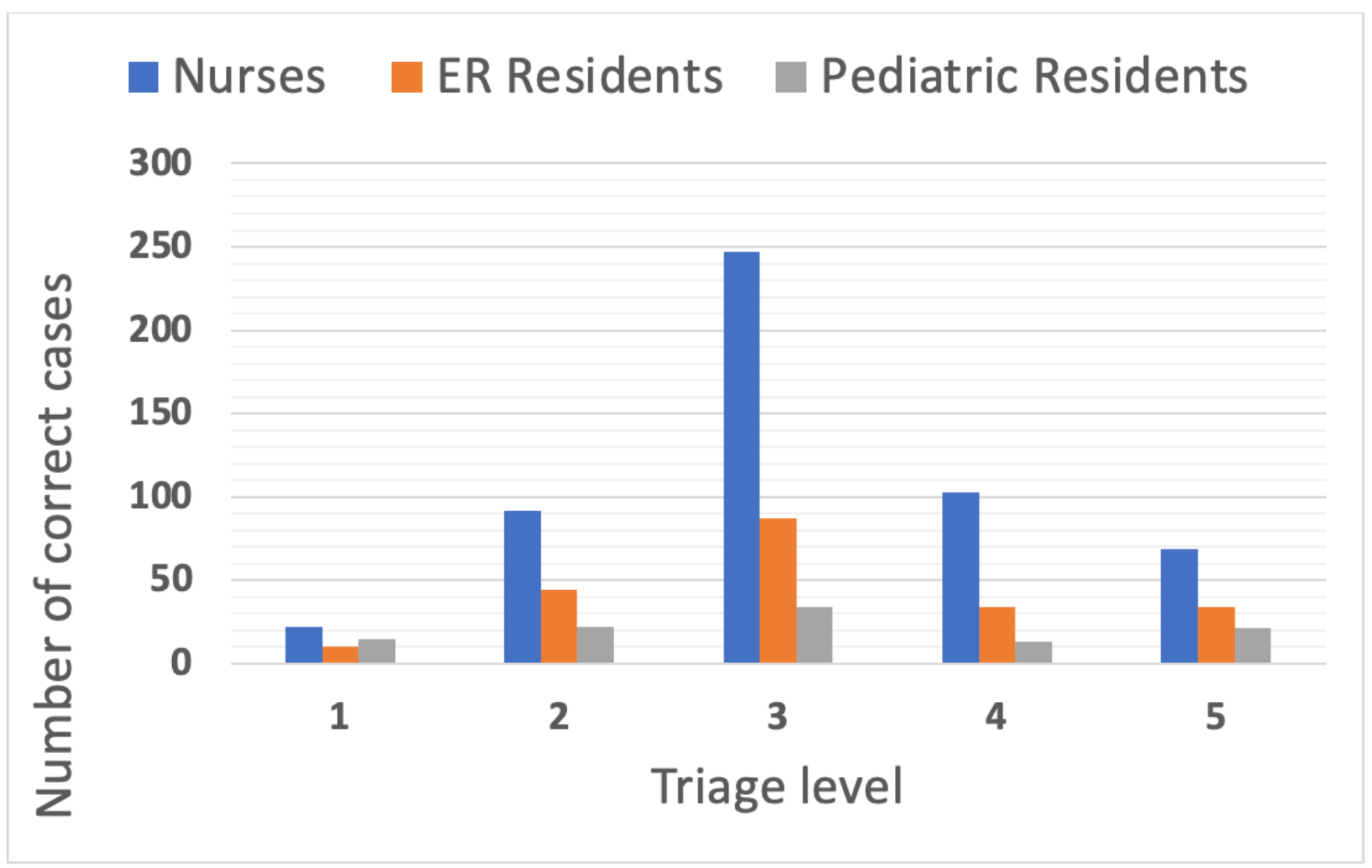




\section{Pairwise Comparisons of Participants}

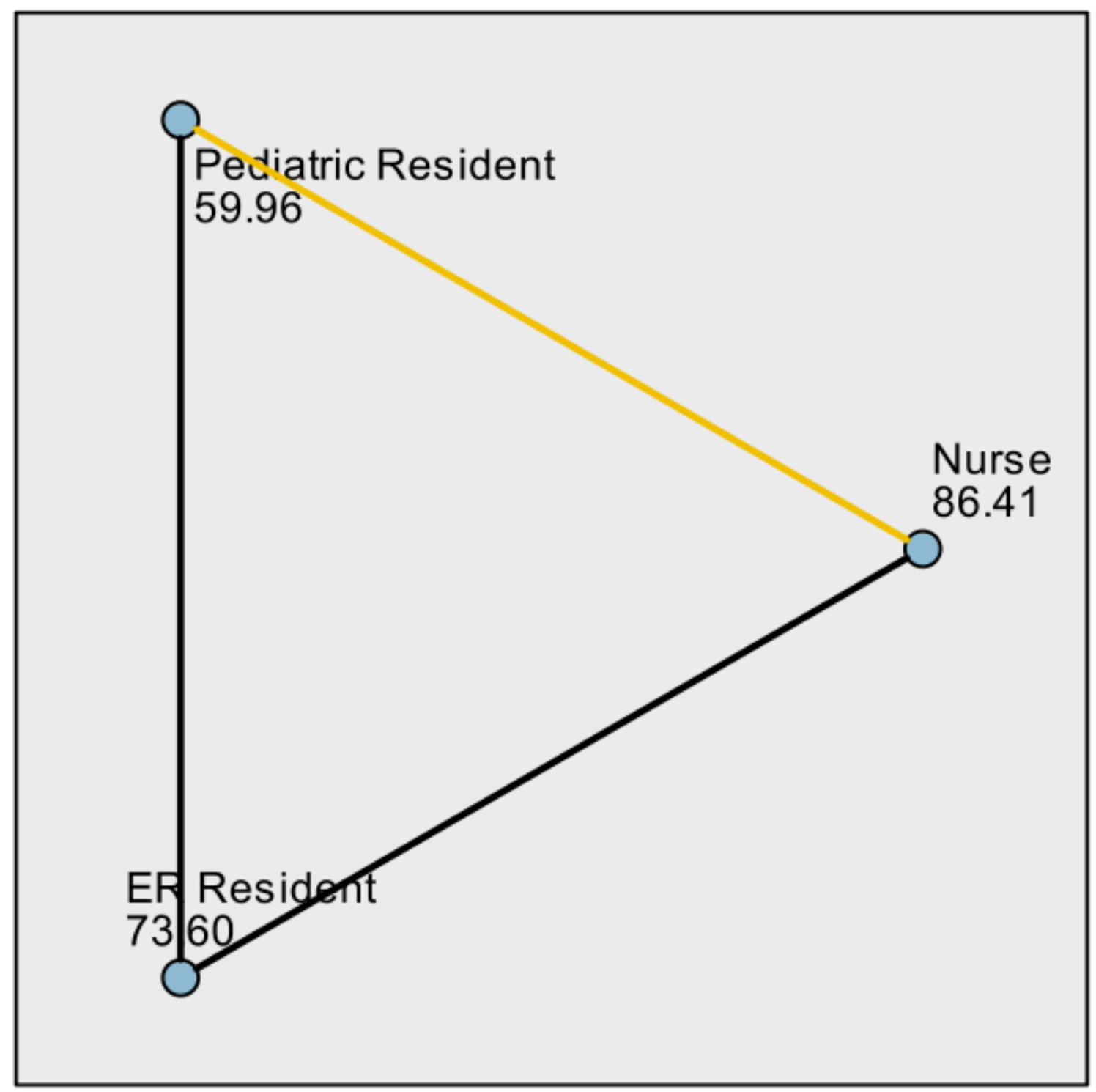

Figure 2

Post Hoc Tests for the triage score and the participants

\section{Supplementary Files}

This is a list of supplementary files associated with this preprint. Click to download. 
- CTASCases.docx

- STROBEchecklistv4combinedPlosMedicine.pdf 